\title{
Krasemann, Benjamin
}

\section{Zur Archivierung von Fällen}

Wittek, Doris [Hrsg.]; Rabe, Thorid [Hrsg.]; Ritter, Michael [Hrsg.]: Kasuistik in Forschung und Lehre. Erziehungswissenschaftliche und fachdidaktische Ordnungsversuche. Bad Heilbrunn : Verlag Julius Klinkhardt 2021, S. 245-258

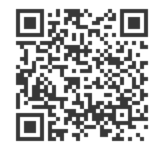

\section{Quellenangabe/ Reference:}

Krasemann, Benjamin: Zur Archivierung von Fällen - In: Wittek, Doris [Hrsg.]; Rabe, Thorid [Hrsg.]; Ritter, Michael [Hrsg.]: Kasuistik in Forschung und Lehre. Erziehungswissenschaftliche und fachdidaktische Ordnungsversuche. Bad Heilbrunn : Verlag Julius Klinkhardt 2021, S. 245-258 - URN: urn:nbn:de:0111-pedocs-215712 - DOI: 10.25656/01:21571

https://nbn-resolving.org/urn:nbn:de:0111-pedocs-215712

https://doi.org/10.25656/01:21571

in Kooperation mit / in cooperation with:

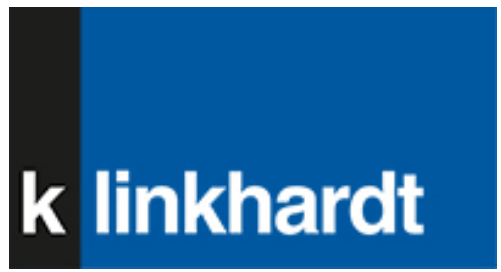

http://www.klinkhardt.de

\section{Nutzungsbedingungen}

Dieses Dokument steht unter folgender Creative Commons-Lizenz: http://creativecommons.org/licenses/by-nc-sa/4.0/deed.de - Sie dürfen das Werk bzw. den Inhalt unter folgenden Bedingungen vervielfältigen, verbreiten und öffentlich zugänglich machen sowie Abwandlungen und Bearbeitungen des Werkes bzw. Inhaltes anfertigen: Sie müssen den Namen des Autors/Rechteinhabers in der von ihm festgelegten Weise nennen. Dieses Werk bzw. der Inhalt darf nicht für kommerzielle Zwecke verwendet werden. Die neu entstandenen Werke bzw. Inhalte dürfen nur unter Verwendung von Die neu entstandenen Werke bzw. Inhalte dürfen nur unter Verwendung von
Lizenzbedingungen weitergegeben werden, die mit denen dieses Lizenzvertrages identisch oder vergleichbar sind.

Mit der Verwendung dieses Dokuments erkennen Sie die Nutzungsbedingungen an.

\section{Terms of use}

This document is published under following Creative Commons-License: http://creativecommons.org/licenses/by-nc-sa/4.0/deed.en - You may copy, distribute and transmit, adapt or exhibit the work in the public and alter, transform or change this work as long as you attribute the work in the manner specified by the author or licensor. You are not allowed to make commercial use of the work. If you alter, transform, or change this work in any way, you may distribute the resulting work only under this or a comparable license.

By using this particular document, you accept the above-stated conditions of use.

\section{Kontakt / Contact:}

\section{peDOCS}

DIPF | Leibniz-Institut für Bildungsforschung und Bildungsinformation Informationszentrum (IZ) Bildung

E-Mail: pedocs@dipf.de

Internet: www.pedocs.de

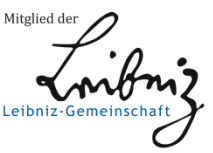




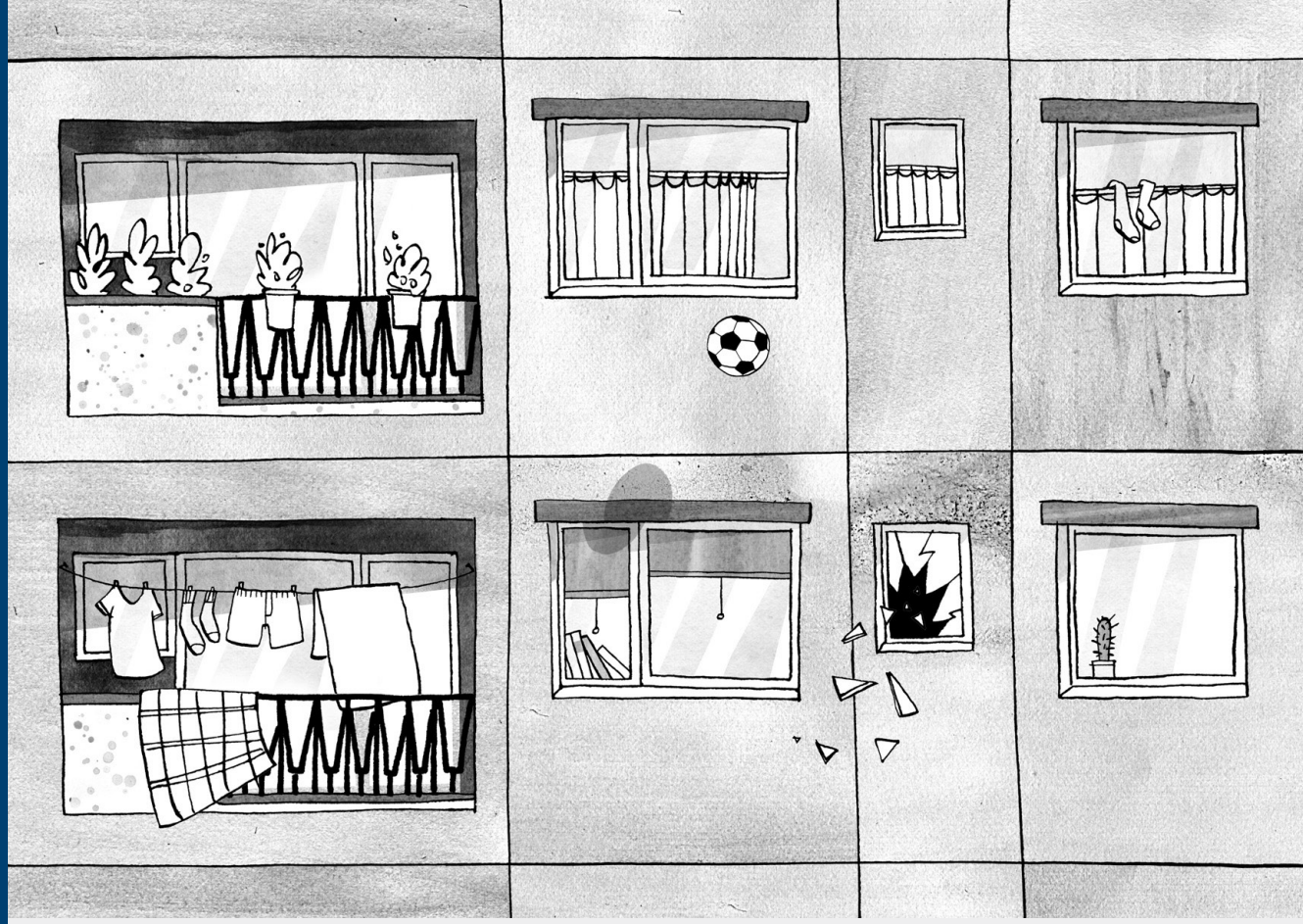

Doris Wittek

Thorid Rabe

Michael Ritter

(Hrsg.)

Kasuistik in Forschung

und Lehre

Erziehungswissenschaftliche und

fachdidaktische Ordnungsversuche 


\section{Doris Wittek Thorid Rabe Michael Ritter (Hrsg.)}

\section{Kasuistik in Forschung und Lehre}

Erziehungswissenschaftliche und fachdidaktische Ordnungsversuche 
Das Projekt KALEI (Kasuistische Lehrerbildung für inklusiven Unterricht), in dessen Rabmen diese Publikation entstanden ist, wird im Rahmen der gemeinsamen „Qualitätsoffensive Lehrerbildung "von Bund und Ländern aus Mitteln des Bundesministeriums für Bildung und Forschung unter dem Förderkennzeichen 01JA1618 gefördert.

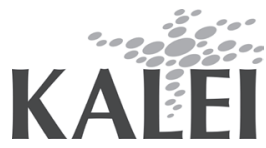

GEFÖRDERT VOM

Bundesministerium

für Bildung

und Forschung

Dieser Titel wurde in das Programm des Verlages mittels eines Peer-Review-Verfahrens aufgenommen. Für weitere Informationen siehe www.klinkhardt.de.

Bibliografische Information der Deutschen Nationalbibliothek Die Deutsche Nationalbibliothek verzeichnet diese Publikation in der Deutschen Nationalbibliografie; detaillierte bibliografische Daten sind im Internet abrufbar über http://dnb.d-nb.de.

2021.ng. () by Julius Klinkhardt.

Zeichnung Umschlagseite 1: () Wolfgang Philippi, Wuppertal (www.wolfgangphilippi.de).

Druck und Bindung: AZ Druck und Datentechnik, Kempten.

Printed in Germany 2021.

Gedruckt auf chlorfrei gebleichtem alterungsbeständigem Papier.

(c) (i)(9) Die Publikation (mit Ausnahme aller Fotos, Grafiken und Abbildungen) ist veröffentlicht unter der Creative Commons-Lizenz: CC BY-NC-SA 4.0 International https://creativecommons.org/licenses/by-nc-sa/4.0/ 


\section{Inhalt}

Doris Wittek, Thorid Rabe, Michael Ritter

Kasuistik in Forschung und Lehre - Ordnungen und Unordnungen

mit Blick auf die Disziplinen

Fälle disziplinär diskutieren - Fälle interdisziplinär verorten

Merle Hummrich

Der Fall aus der Perspektive der Erziehungswissenschaft

Friederike Heinzel

Der Fall aus der Perspektive von Schulpädagogik und

Lehrer*innenbildung: Ein Ordnungsversuch 41

\section{Ursula Bredel, Irene Pieper}

Der Fall aus der Perspektive der Fachdidaktik:

Fachliche Lernprozesse als Ziel und Ausgangspunkt

Jörg Dinkelaker

Der Fall aus der Perspektive der Erwachsenenpädagogik:

Fälle als Grenzobjekte zwischen erziehungswissenschaftlicher und pädagogischer Praxis

Diana Handschke, Bettina Hünersdorf

Der Fall aus der Perspektive der Sozialpädagogik: Fallverstehen als

Kristallisationspunkt zwischen Profession,

Disziplin und dem Politischem 108

\section{Mit Fällen forschen - durch Fälle Wissen schaffen}

\section{Carla Schelle}

Funktionen von Fallarbeit in der Forschung: Fallverständnisse und

Darstellungsweisen aus der Schul- und Unterrichtsforschung

Werner Helsper

Der Fall in der wissenschaftlichen Erkenntnisbildung:

Ein Ordnungsversuch und Konsequenzen für die

Lehrerprofessionalisierung 
6 Inhaltsverzeichnis

Mit Fällen lehren - an Fällen lernen

Richard Schmidt, Doris Wittek

Ziele und Modi von Fallarbeit in der universitären Lehre

Tobias Leonhard

Der Fall in den Schul- bzw. Berufspraktischen Studien

Olaf Krey, Thorid Rabe, Michael Ritter

Fallarbeit in den Fachdidaktiken: Eine analytische Auseinandersetzung

mit Studienelementen der Physik- und Deutschdidaktik

Marcus Syring

Videobasierte Kasuistik in der Lehre

Benjamin Krasemann

Zur Archivierung von Fällen

(Quer-)Perspektiven eröffnen - (Un-)Ordnungen neu denken

Richard Schmidt, Doris Wittek

Rekonstruktive Kasuistik - ein unerreichbares Ideal universitärer Lehre.

Empirische Hinweise zum Widerstreit von Programmatik und Praxis

Thomas Wenzl

Der Fall als Reflexionsübung? Oder: Die erziehungswissenschaftliche

Kasuistik im Lichte der Fallarbeit im Studium der Jurisprudenz und

der Medizin

Andreas Wernet

Fallstricke der Kasuistik

Autor*innenverzeichnis 


\section{Benjamin Krasemann}

\section{Zur Archivierung von Fällen}

\section{Einleitung}

Die Arbeit mit Fällen hat sich in den letzten Jahren in der Lehrer*innenbildung mit unterschiedlichen Entwicklungen, Kontroversen, Diskurssträngen, Begrifflichkeiten, Konzepten und Methoden etabliert (vgl. Schelle 2011, 86; Hebenstreit u.a. 2016, 1). Bereits in den 1980er-Jahren argumentierte Binneberg in der Zeitschrift für Pädagogik für eine kasuistische Pädagogik, mit der „die Theorie und Praxis in der Pädagogik einander wieder nähergebracht werden könnte[...] “ (Binneberg 1979, 397). Wiederhall fand dieses Plädoyer in der Lehrer*innenbildung in vielfacher Art und Weise, unter anderem auch in den KMK-Standards, in denen Fallorientierung als ein Ansatz zur Vermittlung von bildungswissenschaftlichen Inhalten im Lehramtsstudium seit 2004 explizit aufgeführt wird (KMK 2004, 5). Hinzu kommen zahlreiche Veröffentlichungen, die in den letzten Jahren entstanden sind und sich der Arbeit mit Fällen sowohl theoretisch als auch konzeptionell widmen (vgl. exemplarisch Ohlhaver \& Wernet 1999; Ohlhaver 2011; Reh u.a. 2013; Pieper u.a. 2014; Steiner 2014; Hummrich u.a. 2016; Schmidt u.a. 2019). Ein weiterer Indikator für die Prominenz kasuistischer Ansätze in der deutschsprachigen Lehrer*innenbildung ist die Gründung der Arbeitsgemeinschaft Kasuistik in der Lehrer*innenbildung, ${ }^{1}$ die im Jahr 2014 auf einer durch Katharina Kunze und Bernd Stelmaszyk $(\dagger)$ konzipierten und veranstalteten Tagung in Würzburg begonnen wurde.

Seit den frühen 2000er-Jahren finden sich schließlich erste konkrete Bemühungen zum Aufbau (schul-)pädagogischer Fallarchive an deutschen Universitäten. Sie stellen eine Reaktion auf die Forderungen und damit verbundenen Umsetzungsherausforderungen im Kontext von Fallarbeit in der Lehrer*innenbildung dar mit dem Ziel, Material für die Arbeit mit Fällen zur Verfügung zu stellen. Fallmaterial findet sich verstreut über unterschiedliche Veröffentlichungen und entsteht im Kontext von Forschungsprojekten und Seminaren - Fallarchive wurden hier als eine Möglichkeit gesehen, dieses Material zu sammeln, zu systematisieren und zugänglich zu machen. Etwa zeitgleich wurden gleich mehrere Projekte dazu gestartet. An den Universitäten in Frankfurt am Main und Kassel begannen Andreas

1 www.ag-kasuistik.de (11.06.2020) 
Gruschka und Friederike Heinzel online basierte Fallarchive aufzubauen. An der TU Berlin wurde durch Sabine Reh und Kolleg*innen um 2007 die Arbeitsstelle für Pädagogische Kasuistik initiiert, die auch einen Archivansatz beinhaltete.

Mit dem vorliegenden Beitrag ${ }^{2}$ soll ein Überblick über die aktuelle Fallarchiv-Landschaft in der (deutschsprachigen) Lehrer*innenbildung gewonnen werden. Dabei soll den Fragen nachgegangen werden, was Fallarchive leisten können, welche Potenziale sie für die Lehrer*innenbildung haben und welche Herausforderungen hinsichtlich der Ausgestaltung und ihres Betriebs bestehen.

\section{Fallarchive und Fallarbeit - Zur Systematisierung von Fällen}

Bevor ich einige Überlegungen zu derzeit bestehenden Archiven darlege, soll einleitend der Frage nachgegangen werden, was ein Archiv im eigentlichen Sinne ist. Archive lassen sich definieren als „Einrichtungen, deren Aufgabe die systematische Übernahme, Erfassung, Ordnung, dauerhafte Aufbewahrung und Erschließung von Schrift-, Bild- und Tonträgern sowie elektronischer Speichermedien aus öffentlichen Dienststellen, anderen Institutionen (Verbänden, Unternehmen) oder von Einzelpersonen ist" (Reimann 2008, 24). Über einen langen Zeitraum werden in dem spezifischen Format der pädagogischen/erziehungswissenschaftlichen Fallarchive nicht nur Fälle aus dem Kontext Schule und Bildung gesammelt, sondern damit auch die methodischen und konzeptionellen Entwicklungen in diesem Kontext dokumentiert und nachvollziehbar gemacht. Archive im Bereich der pädagogischen Kasuistik, die auch einen forschungsmethodischen Schwerpunkt verfolgen, lassen so, über die Sammlung und Archivierung von Fällen hinaus, die Entwicklungen von Forschungskonventionen und -traditionen erkennbar werden. Das ist möglicherweise auch eine Besonderheit kasuistischer Fallarchive im deutschsprachigen Raum, die überwiegend im Kontext der rekonstruktiven Bildungsforschung und ihrer Methoden verortet sind.

Grundsätzlich stellen Fallarchive eine Arbeitshilfe dar, in der Fallmaterial gesammelt, systematisiert und geordnet wird. In der Regel werden diese Fallarchive heutzutage online angelegt. Die Entstehungskontexte von Fallarchiven sind dabei so unterschiedlich wie das Material, das in ihnen gesammelt wird. Doch wie wird ein Fall zum Material? Ein Fall ist eine Abfolge von Ereignissen oder ein einzelnes konkretes Ereignis, das „nur dann zum, Fall' wird, wenn [es] für mindestens ein erkennendes Subjekt ins Zentrum seiner Aufmerksamkeit rückt. " (Steiner 2014, 8,

2 Der vorliegende Beitrag wurde im Rahmen der gemeinsamen Qualitätsoffensive Lehrerbildung von Bund und Ländern mit Mitteln des Bundesministeriums für Bildung und Forschung unter dem Förderkennzeichen 01JA1505 gefördert. Die Verantwortung für den Inhalt des Beitrags liegt beim Autor. 
H.i.O.). Neben besagten Situationen können auch andere Datensorten, z.B. Biographien, Schulordnungen, Elternbriefe etc. aus dem Kontext Schule und Unterricht, zum Fall gemacht werden. Pädagogische Praxis wird so auf unterschiedlichen Wegen, in der Regel als Text oder Video, zugänglich gemacht zu Lehr- und Lernzwecken oder anderweitiger (wissenschaftlicher) Verwendung. Sowohl die Begriffsfülle als auch die mit Fallarbeit verbundenen Möglichkeiten sind dabei außerordentlich groß und variantenreich (vgl. Tab. 1), demzufolge befindet sich auch sehr unterschiedliches Material in den Fallarchiven.

Wichtige Quellen dafür sind sämtliche Veröffentlichungen, in denen eine pädagogische bzw. schulische Praxis zum Fall gemacht wird bzw. authentische Situationen aus Schule und Unterricht gezeigt werden - das umfasst zunächst alle Formen von Protokollen, Beobachtungen, Situationsbeschreibungen und Transkripten. In Absprache mit Autor*innen und Verlagen können dann Ausschnitte daraus, in der Regel das empirische Material, für die Weiterarbeit genutzt werden. Eine weitere, damit im Zusammenhang stehende Quelle stellen wissenschaftliche Forschungsarbeiten allgemein dar. Oftmals befinden sich in den wissenschaftlichen Veröffentlichungen nur Ausschnitte aus dem gesamten Forschungsmaterial, z.B. eines spezifischen Forschungsprojektes oder einer Qualifikationsarbeit, die beispielhaft Analysen von Fällen als Ausschnitte zeigen. Das im Gesamtkontext entstandene Material verbleibt oft bei den Wissenschaftler*innen - gerade hier findet sich ein Materialschatz, der hohes Potenzial für die Bereitstellung in Fallarchiven bieten kann.

Neben veröffentlichten Texten entsteht Fallmaterial auch in Lehrkontexten, z.B. in Seminaren, in denen Studierende eigene Beobachtungen durchführen und unter unterschiedlichen Anforderungen und Maßgaben etwas zum Fall machen. Gerade im Zusammenhang mit Praxisphasen in der Lehrer*innenbildung und den damit verbundenen, durchaus kritisch zu betrachtenden, Reflexionsangeboten bzw. -aufforderungen entstehen Beobachtungen von Studierenden.

Eine weitere Form stellen, wie bereits erwähnt, reale Dokumente bzw. Materialien aus dem Kontext von Schule und Unterricht dar. Dazu gehören z.B. Elternbriefe, Zeugnistexte, Aufgaben oder Schulordnungen. Auch hier ist die Breite der Möglichkeiten außerordentlich groß. Zu diesen Materialsorten gibt es bisher keine systematischen Fall- bzw. Materialsammlungen.

Von diesen Formen realer Fälle sind schließlich fiktive Fälle zu unterscheiden. Solche fiktiven Fälle können durch Lehrende, Wissenschaftler*innen, aber auch durch Studierende künstlich erzeugt werden, z.B. durch textbasierte und filmisch aufgezeichnete Fallgeschichten oder Fallvignetten, die an der Realität orientierte Situationen aus Schule und Unterricht darstellen bzw. inszenieren. Dabei wird der Fall so angelegt, dass er einem bestimmten Lernziel oder einer bestimmten hochschuldidaktischen Lernumgebung etc. intentional angepasst wird. 
Die in der Tabelle 1 zur Übersicht verwendeten Fallbegriffe werden über die bestehende Literatur zu (pädagogischer) Kasuistik durchaus unterschiedlich und teilweise synonym verwendet. Das gilt insbesondere für englischsprachige Begriffe wie case und case-work. Eine Ursache dafür ist, dass mit diesen Begriffen, ähnlich wie bei den Begriffen Fall und Fallarbeit, nicht nur die Lehre im Mittelpunkt steht, sondern konkret zu bearbeitende Fallsituationen. Im Kontext der sozialen Arbeit weisen sie z.B. direkte Bezüge zu klientenbezogener Arbeit auf.

Tab. 1: Systematisierung von Fallmaterialien und Verwendung im Kontext kasuistischer Lehre, orientiert an Kunze 2016; Grummt 2019; Schmidt u.a. 2019

\begin{tabular}{|c|c|c|c|}
\hline & Fiktiver Fall & \multicolumn{2}{|c|}{ Realer Fall } \\
\hline $\begin{array}{l}\text { Fallarbeit } \\
\text { mit: }\end{array}$ & $\begin{array}{l}\text { Fiktive Fallgeschichte, } \\
\text { fiktive Fallvignette }\end{array}$ & $\begin{array}{l}\text { Falldarstellung, Fall- } \\
\text { vignetten }{ }^{3}, \text { Fallbericht, } \\
\text { case, case-work }\end{array}$ & \begin{tabular}{|l} 
Fallstudie, Fallanalyse, \\
Fallrekonstruktion
\end{tabular} \\
\hline \multirow[t]{3}{*}{ Form } & $\begin{array}{l}\text { Der Fall als „Fall von..." } \\
\text { Text, Erzählung und } \\
\text { Video als Inszenierung }\end{array}$ & $\begin{array}{l}\text { Der Fall als „Fall von..." } \\
\text { Text, Protokoll, Tran- } \\
\text { skript, Videographie, } \\
\text { erzählte Fälle } \\
\end{array}$ & $\begin{array}{l}\text { Der Fall als Analyseeinheit } \\
\text { Text, Protokoll, Transkript } \\
\text { und Videographie }\end{array}$ \\
\hline & $\begin{array}{l}\text { Drehbuch-artige Ab- } \\
\text { läufe, die Probleme } \\
\text { exakt bestimmbar } \\
\text { machen }\end{array}$ & \multicolumn{2}{|c|}{$\begin{array}{l}\text { Darstellung einer auffallenden Situation/eines auffal- } \\
\text { lenden Materials aus der Praxis }\end{array}$} \\
\hline & \multicolumn{2}{|c|}{$\begin{array}{l}\text { Berufsfeldbezogen-handlungsorientierte Reflexion } \\
\text { Subsumtionslogisches Vorgehen, in der Regel } \\
\text { problemerklärend, -darstellend bzw. -lösend }\end{array}$} & $\begin{array}{l}\text { Wissenschaftliche Reflexion/ } \\
\text { Rekonstruktion } \\
\text { > Rekonstruktionslogisches } \\
\text { Vorgehen, in der Regel } \\
\text { problemerschließend bzw. } \\
\text {-analysierend }\end{array}$ \\
\hline $\begin{array}{l}\text { Einsatz- } \\
\text { möglich- } \\
\text { keiten in } \\
\text { der Lehre }\end{array}$ & \multicolumn{2}{|c|}{$\begin{array}{l}\text { - Problembasiertes Lernen } \\
\text { - Case-Based-Reasoning } \\
\text { - Aufgabenbasierte Fallarbeit (zur Erarbeitung } \\
\text { des Falls) } \\
\text { - Kollegiale Fallberatung/-besprechung } \\
\text { - Teile von Portfolio bzw. ePortfolio Angeboten } \\
\text { - Noticing } \\
\text { - Anchored Instruction } \\
\text { - Fall als Teil eines Erhebungsinstruments }\end{array}$} & $\begin{array}{l}\text { - Erlernen von qualitativen } \\
\text { Interpretationsmethoden } \\
\text { z.B. durch aufgabenba- } \\
\text { sierte Fallarbeit (zur Erar- } \\
\text { beitung der Erhebungs- } \\
\text { methode) } \\
\text { - Methodengeleitete Rekon- } \\
\text { struktion von Situationen } \\
\text { aus Schule und Unterricht }\end{array}$ \\
\hline
\end{tabular}

Die Unterscheidung zwischen Fallmaterial als Text und Video ist für die Anlage von online basierten Fallarchiven von großer Bedeutung. Für Fallmaterial, welches als Text vorliegt, muss die Einwilligung der Autor*innen eingeholt sein,

3 Die Unterscheidung zwischen Falldarstellungen und Fallvignetten ist oft fließend. Bei beiden handelt es sich um in sich abgeschlossene Situationsbeschreibungen aus der Praxis. 
sowie die Erlaubnis des Verlages, falls das Material bereits über einen Verlag publiziert ist. Für sämtliche Formen veröffentlichter Protokolle aus dem Kontext von Schule und Unterricht, die z.B. als Ergebnis studentischer Beobachtungsaufgaben entstehen, sollten darüber hinaus die Standards der datenschutzrechtlichen Anforderungen in der empirischen Bildungsforschung gelten (vgl. dazu Meyermann $\&$ Porzelt 2017). Bei bereits veröffentlichtem Material ist diese Voraussetzung in der Regel erfüllt.

Während der Fall als Text relativ einfach in ein Archiv eingestellt und verfügbar gemacht werden kann, sind mit der Archivierung und Zugänglichmachung von Video- und Audioaufzeichnungen weitaus höhere Hürden und Anforderungen verbunden. Diese Regelungen gelten auch für die Veröffentlichungen von Bildern. Entscheidend dabei ist das Recht am eigenen Bild ${ }^{4}$ als Unterpunkt des durch Artikel 2 Absatz 1 in Verbindung mit Artikel 1 Grundgesetz geschützten Rechts auf informationelle Selbstbestimmung. So muss das Einverständnis von Personen vorliegen, die im Video/Bild zu sehen sind oder die man auf Video- und Audioaufzeichnungen hört. In wie weit sich dieses dann auch auf Fallarchive übertragen lässt, muss im Einzelfall entschieden werden. Die schnelle Verfügbarkeit im Kontext von Seminaren ist dabei nur schwer zu gewährleisten und erfordert in der Regel standortgebundene Archivlösungen, z.B. innerhalb eines universitären Netzwerkes oder mit einer regelmäßigen Nutzer*innen-Administration, die mitunter Unterschriften der jeweiligen Nutzer*innen benötigt. In den letzten Jahren sind darüber hinaus videobasierte Fallformate entstanden, in denen drehbuchartige Situationen aus Schule und Unterricht im Film zu einem bestimmten Lehrzweck inszeniert werden (vgl. u.a. Gartmeier 2014) und die nicht diesen Beschränkungen und Regelungen unterliegen.

Nach diesen Überlegungen zur Arbeit mit Fällen und deren Bereitstellung in Archiven soll nun ein Überblick über einige derzeit im deutschsprachigen Raum verfügbare Angebote im Kontext der pädagogischen Kasuistik erfolgen. ${ }^{5}$

\section{3 Überblick über bestehende kasuistische Fallarchive in der Lehrer*innenbildung}

Im deutschsprachigen Raum sind in den letzten Jahren, neben den bereits genannten, eine ganze Reihe von Fallarchiven entstanden, die explizit in der Lehrer*innenbildung verortet sind, aber auch andere an Kasuistik interessierte Personen

4 Auch Bildnisrecht; zu achten ist in dem Zusammenhang auch auf die Vertraulichkeit des Wortes.

5 Als Hauptkriterium der Auswahl diente die aktuelle Abrufbarkeit und Auffindbarkeit des Archivs. Als Datum des Abrufes der Homepages und aller von dort zitierten Informationen wird der 07.12.2019 angegeben. 
adressieren. Bemerkenswert ist, dass obwohl auch im englischsprachigen Raum eine langjährige Tradition in der Arbeit mit Fällen besteht, nach der aktuellen Recherche kein vergleichbares Fallarchiv besteht, welches ähnlich aufgebaut ist, wie die hier vorgestellten Fallarchive. Auch in anderen Disziplinen, wie z.B. der sozialen Arbeit, gibt es bisher keine vergleichbaren Fallarchive.

In der folgenden Übersicht werden Text- und Videoarchive getrennt voneinander betrachtet. Über den Blick auf die sich derzeit in Betrieb befindlichen Portale hinaus werden noch weitere explizit kasuistische Veröffentlichungsformate angesprochen.

\subsection{Textbasierte Fallarchive im deutschsprachigen Raum}

\section{ApaeK-Archiv für pädagogische Kasuistik, Goethe-Universität Frankfurt am Main}

Das bereits zu Anfang dieses Beitrags erwähnte ApaeK wurde durch Andreas Gruschka an der Goethe-Universität Frankfurt am Main gegründet und ist eines der ältesten und größten Fallarchive. Es handelt sich dabei um ein textbasiertes Fallarchiv, das nur mittels eines Zugangsschlüssels abrufbar ist. Die Vergabe erfolgt kurzfristig durch die Mitarbeiter*innen des ApaeK. In der Archivdatenbank befinden sich unterschiedliche Dokumente aus der pädagogischen Praxis sowie Informationen und Handreichungen zu kasuistischem Arbeiten. Für Lehrende besteht die Möglichkeit seminarbezogene Zugangsschlüssel zum Archiv zu erhalten, um so die Verwendung des Archivs in Lehrveranstaltungen zu erleichtern. Neben Fallmaterial bietet das ApaeK „Materialien für die Organisation und Durchführung der Schulpraktischen Studien forschungsbezogenen Typs“. ${ }^{6}$ Es wird explizit darauf verwiesen, dass Materialien, die von Studierenden im Rahmen schulpraktischer Studien erstellt werden, z.B. Unterrichtstranskripte oder andere von Studierenden verfasste Texte, in die Archivdatenbank aufgenommen werden, die dann wieder in Seminaren eingesetzt werden können. Gleichzeitig entsteht damit eine Archivdatenbank für Forscher*innen, die das Material für eigene Forschungsvorhaben nutzen können. Die Fallrecherche ist über unterschiedliche systematisierte Verschlagwortungen und eine mehrdimensionale Suchmaske möglich. Über das angelegte Nutzer*innenprofil können darüber hinaus Favoriten angelegt und die Historie bisher heruntergeladener Dokumente nachvollzogen werden. Link: www.apaek.uni-frankfurt.de

\section{Online-Fallarchiv Schulpädagogik, Universität Kassel}

Das Online-Fallarchiv Schulpädagogik (OFAS) wurde von Friederike Heinzel an der Universität Kassel gegründet. Wie auch das ApaeK ist es eines der weni-

6 https://www.apaek.uni-frankfurt.de/55818490/F\%C3\%BCr_Dozenten (28.09.2020) 
gen onlinebasierten Projekte, das mittlerweile auf ein über zehnjähriges Bestehen zurückblicken kann. Bei diesem Archiv-Projekt ist der Fallbestand zum großen Teil frei zugänglich und keine Anmeldung nötig. Deshalb sind im OFAS auch keine Nutzer*innenprofile vorgesehen. Bei passwortgeschützten Fällen muss das Passwort via Mail angefordert werden. Die Bearbeitung erfolgt kurzfristig durch Mitarbeiter*innen des OFAS. Der Fallbestand umfasst zum überwiegenden Teil bereits veröffentlichtes Material. Dabei werden die Textausschnitte grundsätzlich mit einer Verlinkung zum Originaltext auf der jeweiligen Verlagsseite zur Verfügung gestellt. Damit besteht die Möglichkeit, den Gesamtkontext des Fallmaterials oder den Forschungszusammenhang nachzulesen. Recherchiert werden kann nach Autor*innen, Interpretationsmethoden, Fachdidaktiken und Schulformen. Zusätzlich kann eine Freitext- und Schlagwortsuche genutzt werden. Im OFAS befinden sich außerdem Lehrtexte und Lehrfilme zu qualitativen Interpretationsmethoden sowie konkrete Arbeitshilfen zur Arbeit mit Fällen. Für die Arbeit mit Fällen aus dem Archiv werden Aufgabenformate angeboten (aufgabenbasierte Fallarbeit) sowie ein Modell der kollegialen Fallbesprechung, das sich an Abläufen der kollegialen Fallberatung orientiert und für die Arbeit mit fremden und eigenen Fällen im Seminarkontext angepasst wurde. Neben diesen subsumtionslogischen Zugängen wird auch ein rekonstruktionslogisches Verfahren als fester Ablauf für die Arbeit mit Fällen in Seminaren vorgeschlagen. Sequentielles Interpretieren orientiert sich dabei an einem Teilschritt von Rekonstruktionsverfahren wie der Objektiven Hermeneutik, der Interaktionsanalyse oder der Dokumentarischen Methode. Wie auch das Fallmaterial und die Lehrtexte können die Arbeitsvorschläge als PDF heruntergeladen werden. In Planung befindet sich ein Recherche-Bereich für durch Studierende verfasste Situationsbeschreibungen. Seit 2017 verwaltet das OFAS zudem den kompletten Forschungsdatensatz, der im Projektnetz INTAKT (Soziale Interaktionen in pädagogischen Arbeitsfeldern) entstanden ist. Der INTAKT-Datensatz besteht aus einer unabgeschlossenen Sammlung von Beobachtungsprotokollen aus Schulen sowie aus vor- und außerschulischen Einrichtungen und wird nach Absprache mit dem OFAS zur Verfügung gestellt. Link: www.fallarchiv.uni-kassel.de

\section{sportdidaktik; fallarchiv, Pädagogische Hochschule FHNW Basell Pädagogische Hochschule Freiburg}

Das Projekt sportdidaktik; fallarchiv wurde in der Schweiz von Roland Messmer und Ilka Lüsebrink initiiert. Dabei bestehen sowohl die Möglichkeit einer Freitextsuche, als auch festgelegte Kategorien als Suchangebote. Das Fallarchiv hat mit dem Bezug zur Sportdidaktik eine klare fachliche Ausrichtung. Im Archiv befinden sich ausschließlich Textfälle, zu denen teilweise auch Interpretationen vorliegen. Es handelt sich durchweg um Fallmaterial, das nicht aus bestehenden 
Veröffentlichungen entnommen wurde. Wie auch beim OFAS ist für die Recherche im Fallbestand keine vorherige Anmeldung notwendig.

Link: www.sportdidaktik.ch/fallarchiv

\section{KASUS - Kasuistische Fallsammlung}

Auch das Institut für Erziehungswissenschaft der Universität Hannover verfügt über ein eigenes Fallarchiv. Gesammelt werden hier Transkripte von Studierenden, die unter anderem nach Institutionen, Schlagworten, Materialart, Schulfach, -form und Klassenstufe recherchiert werden können. Das Archiv steht in engem Zusammenhang mit dem Lernangebot am Institut für Erziehungswissenschaft der Universität Hannover. Das Material kommt vor allem von Studierenden und wird über ein Formblatt beim Archiv eingereicht. Das Fallmaterial selbst besteht zu einem großen Teil aus kurzen Sequenzen bzw. Interaktionsprotokollen ohne eine dazugehörige Interpretation.

Link: www.kasus.uni-hannover.de

\section{Fallportal: KALEI - Kasuistische Lehrerbildung für den inklusiven Unterricht} Im Rahmen der Qualitätsoffensive Lehrerbildung wurde auch an der Martin-Luther Universität Halle-Wittenberg ein eigenes Fallarchiv eingerichtet. Dabei hat die Fallsammlung des Fallportals eine klare inhaltliche Ausrichtung. Fallarbeit wird als „ein wichtiger Baustein für die Verknüpfung von inklusionsorientierter Theorie und Praxis"7 im Kontext des Lehramtstudiums verortet. Dafür werden im Archiv sowohl Fälle zur Verfügung gestellt, als auch methodische Hinweise in kurzen Videoclips gegeben, wie (Fall-)Material erhoben und ausgewertet werden kann. In einem kurzen Video wird eine Einführung darüber gegeben, was Kasuistik ist und wie mit Fällen gearbeitet werden kann. Der Zugang zum Fallarchiv selbst erfolgt ohne Anmeldung. Für die Fallrecherche steht eine Freitextsuche zur Verfügung sowie die Möglichkeit, nach Arbeitsfeldern, Themenschwerpunkten und methodischen Einordnungen zu filtern. Teilweise sind Interpretationen zum Fall vorhanden. Darüber hinaus steht Videomaterial aus Sekundärquellen, z.B. im Internet veröffentlichten Videos aus dem Bereich Schule und Unterricht, zur Verfügung.

Link: https://blogs.urz.uni-halle.de/fallarchiv2/

\subsection{Videobasierte Fallarchive im deutschsprachigen Raum}

Bei fast allen videobasierten Fallarchiven sind die Hürden zur Nutzung des Materials relativ hoch. So ist die Nutzung in einigen Fällen nur mit vorheriger Unterschrift möglich oder teilweise in Kombination mit SSO-Anmeldeverfahren (Single Sign-on Verfahren, die eine einmalige Anmeldung mit Authentifizierung

7 https://blogs.urz.uni-halle.de/fallarchiv2/ (28.09.2020) 
mit lokalen Zugriffsberechtigungen erlauben) nur im Netzwerk eines Standortes erlaubt.

\section{Unterricht unter der Lupe, Universität Kassel}

Das Projekt Unterricht unter der Lupe wendet sich sowohl an Dozierende als auch Studierende der Universität Kassel. Es werden Videos zur Verfügung gestellt, die ganze Unterrichtsstunden oder einzelne Unterrichtssequenzen zeigen. Eine Besonderheit ist hier, dass ein großer Teil des Materials aus mehreren Kameraperspektiven aufgenommen wurde, z.B. sind Schüler*innen- und Lehrer*innenperspektiven separat sichtbar. Zudem finden sich Transkriptionen zu den Videos. Begonnen wurde außerdem mit dem Einsatz von 360-Grad-Kameras. Bisher ist der Zugang nur mit einem Nutzer*innenaccount der Universität Kassel möglich. In Ausnahmen kann über eine Anfrage auch ein externer Zugriff erfolgen. Perspektivisch ist eine Erleichterung für die Nutzung außerhalb der Universität Kassel geplant.

Link: www.uni-kassel.de/go/unterricht-unter-der-lupe

\section{Fallarchiv HILDE, Stiftung Universität Hildesheim}

Das Fallarchiv HILDE der Universität Hildesheim wendet sich an alle Akteur*innen der Lehrer*innenbildung sowie an Wissenschaftler*innen aus der Schul-, Unterrichts- und Professionsforschung. Die Unterrichtsaufzeichnungen und die dazugehörigen Begleitmaterialien sind dabei entweder in physischer Form im HILDE Büro ausleihbar oder können über das Portal HILDEonline auch von externen Nutzer*innen abgerufen werden. Neben Videos werden auch hier Unterrichtsmaterialien und Transkripte zu den Videos zur Verfügung gestellt. Externe Nutzer*innen müssen sich dabei zunächst auf einem Streaming Server der Universität Hildesheim anmelden. Das Projekt HILDE bzw. deren Mitarbeiter*innen stellen zudem ihre Expertise bei der Erstellung und Aufbereitung von Unterrichtsvideos zur Verfügung.

Link: www.uni-hildesheim.de/celeb/projekte/fallarchiv-hilde/das-fallarchiv/hildeonline/

\section{unterrichtsvideos.ch, Universität Zürich, Pädagogische Hochschule Schwyz}

Das Videoportal unterrichtsvideos.ch stellt Videomaterial aus unterschiedlichen Fächern und Schulstufen zur Verfügung. Eine Besonderheit dabei ist, dass auch Videos aus anderen Ländern, z.B. Japan oder den USA, abgerufen werden können. Ein kleiner Teil der Videos kann ohne vorherige Anmeldung über die Seite gestreamt werden. Zu den Videos werden teilweise Transkripte und Unterrichtsmaterialien als PDF zur Verfügung gestellt. Die Videos stammen fast ausschließlich aus mehreren Large-Scale-Videostudien des Instituts für Erziehungswissenschaft der Universität Zürich und dem Projekt ICT (Informations- und Kommunikati- 
onstechnologien) im Primarschulunterricht. Neben den Videos werden auch Arbeits- und Verwendungsmöglichkeiten der Videos aufgezeigt.

Link: www.unterrichtsvideos.ch

\section{ViU: Early Science - Videobasierte Unterrichtsanalyse, Universität Münster}

Für die Nutzung des Videomaterials des Münsteraner Videoportals $V i U$ ist eine vorherige Anmeldung mit einer Single Sign-on-fähigen Nutzer*innenkennung notwendig. Betrieben wird das Portal durch das Institut für Psychologie in Bildung und Erziehung, das Seminar für die Didaktik des Sachunterrichts und den ZIV Servicepunkt Film der Westfälischen Wilhelms-Universität Münster. Die gesammelten Videoaufzeichnungen stammen aus dem naturwissenschaftlichen Grundschulunterricht und dienen der Aus- und Weiterbildung von Lehrer*innen. Sie umfassen Videos zur Klassenführung, Lernunterstützung und Szenen mit Anregungen zur Analyse der Lernunterstützung. Die Videos bieten unterschiedliche, synchron aufgezeichnete Kameraperspektiven. Als PDF können zusätzlich der Unterrichtsentwurf, das Schüler*innenmaterial, das Verlaufsprotokoll und das dazugehörige Transkript heruntergeladen werden, wobei diese auch ohne Login bzw. Zugriff auf die Videos genutzt werden können.

Link: www.uni-muenster.de/Koviu/filme/index.html

\section{FOCUS Videoportal, Freie Universität Berlin}

Im FOCUS Videoportal (Fokussierte computerbasierte Unterrichtsanalysen im Studium) der FU Berlin können Unterrichtsvideos und Interviews mit Lehrer*innen und Schüler*innen abgerufen werden. Zu den Videos werden Begleitmaterialien wie Transkripte und Unterrichtsverlaufspläne zur Verfügung gestellt. Daneben gibt es Lehr-Lerngelegenheiten, mit denen die Videos und Begleitmaterialien in didaktisch aufbereiteten Umgebungen systematisiert werden. Eine Toolbox enthält zudem Empfehlungen zu Fachliteratur und Instrumenten der Schul- und Unterrichtsentwicklung. Für die Nutzung von FOCUS sind in allen Bereichen des Angebots eine Nutzer*innenregistrierung und ein anschließender Login notwendig.

Link: www.tetfolio.fu-berlin.de/tet/focus

\subsection{Fallbezogene Veröffentlichungsreihen}

Abschließend zur Übersicht über kasuistische Fallarchive soll noch auf Zeitschriften- und Buchreihen aufmerksam gemacht werden, die ebenfalls einen expliziten kasuistischen Bezug aufweisen. Auch in diesen Reihen finden sich unterschiedliche mit Fallarbeit verbundene Perspektiven sowohl auf den Fall als auch auf methodische Aspekte, z.B. Interpretationsmethoden.

Seit 2015 wird an der Leibniz Universität Hannover die Reihe falltiefen herausgegeben. Hier werden regelmäßig Beiträge von Dozierenden und Studierenden 
veröffentlicht, die im Rahmen kasuistischer Lehrveranstaltungen am IfE der Universität Hannover vor allem im Arbeitsbereich Fallrekonstruktive Schul- und Unterrichtsforschung entstanden sind. Die Zeitschrift wird als Werkschau verstanden, die die kasuistische Arbeit im Kontext der Lehrveranstaltungen sichtbar macht. Die bisherigen, jährlich erscheinenden, Ausgaben sind als PDF kostenlos auf der Homepage der Zeitschrift erhältlich. ${ }^{8}$

Ebenfalls beteiligt ist der Arbeitsbereich an der Buchreihe Pädagogische Fallanthologie, die seit 2008 im Verlag Barbara Budrich erscheint. Herausgegeben wird sie durch Sabine Reh, Andreas Gruschka und Andreas Wernet. In der Reihe erschienen bisher 13 Bände mit Fallanalysen aus unterschiedlichen schulpädagogischen Kontexten.

Eine noch relativ neue Veröffentlichungsreihe ist mit dem Fallarchiv Kindheitspädagogische Forschung (FalKi) entstanden. Das FalKi ist eine Online-Zeitschrift zu qualitativen Methoden in Forschung und Lehre, die durch Stefanie Bischoff-Pabst, Sabine Bollig, Peter Cloos, Iris Nentwig-Gesemann und Marc Schulz herausgegeben wird. Ebenfalls beteiligt ist das Kompetenzzentrum Frühe Kindheit Niedersachsen sowie der Forschungsschwerpunkt Bildungsräume in Kindheit und Familie der TH Köln. Im Mittelpunkt der Veröffentlichungsreihe steht dabei vor allem die Methodenausbildung. Materialien aus qualitativen Forschungsprojekten werden gezeigt, an denen der Forschungsprozess nachvollziehbar dargestellt werden kann. Abschließend soll noch auf eine 2019 gegründete Veröffentlichungsreihe hingewiesen werden, die zwar nicht explizit kasuistisch angelegt ist, aber trotzdem aus dieser Perspektive interessantes Material bzw. Beiträge enthalten kann. Mit datum\& diskurs ${ }^{9}$ wurde ein Veröffentlichungsformat geschaffen, in dem die empirische Wirklichkeit zum Ausgangspunkt erziehungswissenschaftlicher Theoriebildung gemacht wird. Ziel dieses Formats ist dabei die qualitative empirische Auseinandersetzung mit unterschiedlichem Datenmaterial. Das Material, vom Herausgeber*innenteam als Datum bezeichnet, ist demzufolge Ausgangspunkt der dann veröffentlichten Beiträge. Unterschiedliche Autor*innen perspektivieren das identische Datum aus ihren unterschiedlichen methodischen/methodologischen bzw. theoretischen Perspektiven. Aus einer kasuistischen Perspektive kann dieses Format einen besonderen Gewinn dadurch darstellen, dass, wenn man das Datum als Fall betrachtet, daran unterschiedliche analytische Bezüge und Rekonstruktionen aufgezeigt und in einen gemeinsamen Diskurs überführt werden können.

8 https://www.iew.uni-hannover.de/de/forschung/arbeitsbereich-schul-und-professionsforschung/ falltiefen/ (11.06.2020)

9 www.datumunddiskurs.de (11.06.2020) 


\section{Zusammenfassung und Ausblick: Möglichkeiten und Her- ausforderungen für Fallarchive in der Lehrer*innenbildung}

Mittlerweile gibt es ein breites Angebot kasuistischer Fallarchive, die in der Lehrer*innenbildung Verwendung finden. Für die Lehrer*innenbildung weisen sie unterschiedliche Potenziale auf. So können Fallarchive eine Hilfestellung in der wissenschaftlichen Methodenausbildung bieten. Insbesondere Noviz*innen finden hier Möglichkeiten, die Sprache der Interpretation in ihrer Vielfalt qualitativer Forschungsmethoden zu lernen sowie Kenntnisse darüber zu erlangen, was ein Fall ist und in welchen unterschiedlichen methodischen Rahmungen dieser verfasst ist. Auch Wissenschaftler*innen finden hier Material, das unter Umständen für eigene Forschungen genutzt werden kann oder zu dem Sekundäranalysen verfasst werden können. Als Lehr- bzw. Lerngegenstand können Fälle in der Lehrer*innenbildung dazu beitragen, einen reflektierten Rückgriff auf erweiterte „Deutungsrepertoires“ (Bauersfeld 1999, 205), im Sinne einer „fallverstehenden Reflexivität“ (Reh \& Schelle 2019, 14; dazu auch Heinzel \& Krasemann 2015), anzubahnen. Schmidt u.a. sehen das übergeordnete Ziel von Fallarbeit in der Professionalisierung ,angehender Lehrpersonen, also vor allem die Entwicklung professioneller Haltungen, professionellen Wissens oder die Unterstützung professionellen (zukünftigen) Handelns" $(2019,2)$.

Die Hauptfunktion der meisten Archive liegt darin, Lehr- und Lernmaterial bereitzustellen, mit dem authentische Fälle aus der Praxis thematisiert werden können, z.B. in Lehrveranstaltungen. Die digitale Bereitstellung geht in den meisten Fallarchiven einher mit der Option Daten auszudrucken und so entsprechend on paper einsetzen zu können. Mit Blick auf die Entwicklungen der zurückliegenden Jahre zeigt sich zudem, dass der Umfang der Möglichkeiten des Einsatzes mit den technischen Entwicklungen steigt. Diese reichen über das Anlegen von Such- bzw. Recherchefunktionen bis zur Verbindung mit digital basierten Learning Management Systemen. Insbesondere im Bereich videobasierter Daten ist es inzwischen möglich, mehrere Kameraperspektiven einer Situation anzubieten, teilweise in 360 Grad Perspektiven.

Über die Nutzung solcher Archive lassen sich bisher nur schwer Aussagen treffen. Es gibt keine Zahlen darüber, wie viele Personen Fallarchive nutzen und zu welchem Zweck sie dies tun. Klickzahlen geben dabei nur relativ wenig Auskunft darüber, ob und wie die Fälle konkret verwendet werden.

Der hier vorgestellte Überblick über Fallarchive und deren konzeptionelle Besonderheiten zeigt, dass der Aufbau eines solchen Archivs ein intensiver Arbeitsprozess ist. Neben der Konzeption und dem Aufbau müssen dabei auch die fortwährende Pflege und Administration berücksichtigt werden. Grundsätzlich stellt sich die Frage, ob der Aufwand des Neuaufbaus eines Archivs lohnt oder nicht die Zusammenarbeit mit bestehenden Archiven überlegt werden sollte. Fallarchive 
entstehen häufig im Kontext von Drittmittelprojekten mit der für solche Projekte typischen Befristungspraxis des wissenschaftlichen Personals. Für die Anlage solcher und ähnlicher Projekte ist das durchaus folgenreich. So haben Haug und Wedekind (2009) eine Vielzahl von E-Learning-Projekten untersucht, die seit 1999 in einer Höhe von 300 Millionen Euro durch das BMBF gefördert wurden. Nur etwa ein Drittel dieser Projekte war nach etwa neun Jahren im Internet noch auffindbar, in sehr unterschiedlichen Betriebszuständen, die von einer erkennbaren weiterhin bestehenden Administration bis hin zu toten Webseiten reichte. Was die Nachhaltigkeit solcher aufwändig aufgebauten und geförderten Projekte angeht, sei die Befundlage ernüchternd (vgl. Haug \& Wedekind 2009, 32). Zwar handele es sich hierbei um Zahlen sehr unterschiedlicher Projekte und Förderprogramme, trotzdem weisen sie auf Probleme der Handhabung unter Umständen komplexer Fallarchivumgebungen hin. Dies betrifft sowohl die inhaltliche als auch die technische Administration. Datenmaterial muss gesucht, gesichtet und redaktionell bearbeitet werden. Viele Archive bieten darüber hinaus weitere Inhalte an, z.B. Lernpfade, Aufgaben zu Fällen oder Methodenlehrtexte. Die zumeist online basierten Fallarchive benötigen je nach Funktionsumfang eine regelmäßige Administration der technischen Basis. Unabhängig von der gewählten technischen Plattform muss mit regelmäßigen Updateprozessen gerechnet werden. Mitunter verändert sich auch die Webnutzung an sich, z.B. wie in den letzten Jahren durch die Einführungen von Tablets oder Smartphones, für deren Anzeige dann wiederum die Plattform angepasst werden sollte. Handelt es sich bei dem eingestellten Material um solches, das eine Nutzer*innenanmeldung benötigt, kann diese zwar in der Regel automatisiert erfolgen, benötigt aber auch hier eine permanente Kontrolle, z.B. im Rahmen technischer Updates im Backend eines Archivs. Nachhaltig kann das nur gewährleistet werden, wenn das vorhandene Know-How bewahrt, weiterentwickelt und strukturell abgesichert wird (ebd., 35).

\section{Literaturverzeichnis}

Bauersfeld, H. (1999): Fallstudien in der Lehrerausbildung - wozu? In: F. Ohlhaver \& A. Wernet (Hrsg.): Schulforschung - Fallanalyse - Lehrerbildung. Diskussionen am Fall. Opladen: Leske + Budrich, 191-207.

Binneberg, K. (1979): Pädagogische Fallstudien. Ein Plädoyer für das Verfahren der Kasuistik in der Pädagogik. In: Zeitschrift für Pädagogik 25, 395-402.

Gartmeier, M. (2014): Fiktionale Videofälle in der Lehrerinnen- und Lehrerbildung. In: Beiträge zur Lehrerinnen- und Lehrerbildung 32 (2), 235-246.

Grummt, M. (2019): Das hallesche Fallportal - Konzeption, Perspektiven und Möglichkeiten. In: C. Klektau, S. Schütz \& A.J. Fett (Hrsg.): Heterogenitätssensibilität durch Fallarbeit fördern. Zum Stellenwert von Kasuistik und Inklusion in der Lehrer*innenbildung. Halle (Saale): Martin-Luther-Universität Halle-Wittenberg, Zentrum für Lehrer*innenbildung, 99-107.

Haug, S. \& Wedekind, J. (2009): Adresse nicht gefunden. Auf den digitalen Spuren der E-Teaching-Förderprojekte. In: D. Ullrich (Hrsg.): E-Learning: eine Zwischenbilanz. Kritischer Rück- 
blick als Basis eines Aufbruchs. Münster u.a.: Waxmann, 19-38. Online unter: http://www.waxmann.com/fileadmin/media/zusatztexte/2172Volltext.pdf. (Abrufdatum: 18.09.2019).

Hebenstreit, A., Hinrichsen, M., Hummrich, M. \& Meier, M. (2016): Einleitung - eine Reflexion zur Fallarbeit in der Erziehungswissenschaft. In: A. Hebenstreit, M. Hinrichsen, M. Hummrich \& M. Meier (Hrsg.): Was ist der Fall? Kasuistik und das Verstehen pädagogischen Handelns. Wiesbaden: Springer VS, 1-13.

Heinzel, F. \& Krasemann, B. (2015): Lehrerbildung mit dem Online-Fallarchiv Schulpädagogik. In: B. Berendt, A. Fleischmann, N. Schaber \& B. Szczyrba (Hrsg): Neues Handbuch Hochschullehre. Berlin: Raabe, 43-67.

Hummrich, M., Hebenstreit, A., Hinrichsen, M. \& Meier, M. (Hrsg.) (2016): Was ist der Fall? Kasuistik und das Verstehen pädagogischen Handelns. Wiesbaden: Springer VS.

Kunze, K. (2016): Ausbildungspraxis am Fall. Empirische Erkundungen und theoretisierende Überlegungen zum Typus einer praxisreflexiven Kasuistik. In: M. Hummrich, A. Hebenstreit, M. Hinrichsen \& M. Meier(Hrsg.): Was ist der Fall? Kasuistik und das Verstehen pädagogischen Handelns. Wiesbaden: Springer VS, 97-121.

KMK - Sekretariat der Ständigen Konferenz der Kultusminister der Länder in der Bundesrepublik Deutschland (2004): Standards für die Lehrerbildung: Bildungswissenschaften. Beschluss der Kultusministerkonferenz vom 16.12.2004. Online unter: http://www.kmk.org/¿leadmin/veroeffentlichungen_beschluesse/2004/2004_12_16-Standards-Lehrerbildung.pdf. (Abrufdatum: 18.09.2019).

Meyermann, A. \& Porzelt. M. (2017): Datenschutzrechtliche Anforderungen in der empirischen Bildungsforschung- eine Handreichung. In: forschungsdaten bildung informiert 6 (1). Online unter: https://www.forschungsdaten-bildung.de/get_files.php?action=get_file\&file=fdb-informiert-nr-6. pdf. (Abrufdatum:17.09.2019).

Ohlhaver, F. (2011): Fallanalyse, Professionalisierung und pädagogische Kasuistik in der Lehrerbildung. In: Sozialer Sinn 12, 279-303.

Ohlhaver, F. \& Wernet, A. (1999): Zwischen Pädagogik und Erziehungswissenschaft: Ansätze zur systematischen Begründung eines interpretativ-fallanalytischen Vorgehens in der Lehrerbildung. In: F. Ohlhaver \& A. Wernet (Hrsg.): Schulforschung Fallanalyse Lehrerbildung. Diskussionen am Fall. Opladen: Leske + Budrich, 11-28.

Pieper, I., Frei, P., Hauenschild, K. \& Schmidt-Thieme, B. (2014): Was der Fall ist. Beiträge zur Fallarbeit in Bildungsforschung, Lehramtsstudium, Beruf und Ausbildung. Wiesbaden: Springer VS.

Reh, S. \& Schelle, C. (2019): Der Fall im Lehrerstudium - Kasuistik und Reflexion. In: C. Schelle, K. Rabenstein \& S. Reh (Hrsg.): Unterricht als Interaktion. Ein Fallbuch für die Lehrerbildung. Bad Heilbrunn: Klinkhardt, 13-20.

Reh, S., Geiling, U. \& Heinzel, F. (2013): Fallarbeit in der Lehrerbildung. In: B. Friebertshäuser, A. Langer \& A. Prengel (Hrsg.): Handbuch Qualitative Forschungsmethoden in der Erziehungswissenschaft. 2. Auflage, Weinheim und Basel: Beltz Juventa, 911-924.

Reimann, N. (2008): Grundfragen und Organisation des Archivwesens. In: N. Reimann (Hrsg.): Praktische Archivkunde. Ein Leitfaden für Fachangestellte für Medien- und Informationsdienste. Fachrichtung Archiv. Im Auftrag des LWL-Archivamtes für Westfalen. 2. Auflage, Münster: Ardey-Verlag, 23-53.

Schelle, C. (2011): Fallarbeit in der Lehrerbildung - Strukturmerkmale schulischer und unterrichtlicher Interaktion. In: Erziehungswissenschaft 22 43, 85-92.

Schmidt, R., Becker, B., Grummt, M., Haberstroh, M., Lewek, T. \& Pfeiffer A. (2019): Vorschlag für eine Systematisierung kasuistischer Lehr-Lern-Formate in der universitären Lehrer*innenbildung. Online unter: https://blogs.urz.uni-halle.de/fallarchiv2/files/2019/02/KALEI_AK-Kasuistik_Systematisierung-von-Kasuistik.pdf. (Abrufdatum: 11.12.2019).

Steiner, E. (2014): Kasuistik - ein Fall für angehende und praktizierende Lehrpersonen. In: Beiträge zur Lehrerinnen- und Lehrerbildung 32 (1), 6-21. 